\title{
RECK Is Up-Regulated and Involved in Chondrocyte Cloning in Human Osteoarthritic Cartilage
}

\author{
Tokuhiro Kimura, ${ }^{*}$ Aiko Okada, ${ }^{*}$ Taku Yatabe, ${ }^{\star \dagger}$ \\ Masashi Okubo, ${ }^{* \dagger}$ Yoshiaki Toyama, ${ }^{\dagger}$ \\ Makoto Noda, ${ }^{\ddagger}$ and Yasunori Okada* \\ From the Departments of Pathology, and Orthopedic Surgery, \\ School of Medicine, Keio University, Tokyo; and the Department \\ of Molecular Oncology, Kyoto University Graduate School of \\ Medicine, Kyoto, Japan
}

Reversion-inducing cysteine-rich protein with Kazal motifs (RECK) is a membrane-anchored matrix metalloproteinase regulator, but its functions in cartilage are not fully understood. The aim of the present study was to examine the expression and functions of RECK in human osteoarthritic (OA) cartilage. Quantitative RT-PCR indicated that the expression level of RECK is significantly higher in OA cartilage than in normal cartilage. By immunohistochemical analysis, RECK was localized to chondrocytes in OA cartilage, and the immunoreactivity directly correlated with the Mankin score and degree of chondrocyte cloning and proliferation. In cultured OA chondrocytes, RECK was expressed on the cell surface by glycosylphosphatidylinositol anchoring. The expression was stimulated by insulin-like growth factor- 1 and suppressed by interleukin- 1 and tumor necrosis factor- $\alpha$. Down-regulation of RECK by small interfering RNA showed reduced spreading and smaller focal adhesions in the chondrocytes. Chondrocyte migration in a monolayer wounding assay was increased by down-regulation of RECK and inhibited by RECK overexpression in an matrix metalloproteinase activity-dependent manner. On the other hand, chondrocyte proliferation was suppressed by RECK silencing, and this was associated with reduced phosphorylation of focal adhesion kinase and extracellular signal-regulated kinase, whereas the proliferation was enhanced by RECK overexpression. These data are the first to demonstrate that RECK is up-regulated in human OA cartilage and suggest that RECK plays a role in chondrocyte cloning probably through suppression and promotion of chondrocyte migration and proliferation, respectively. (Am J Pathol 2010, 176:2858-2867; DOI: 10.2353/ajpath.2010.091003)
Articular cartilage is composed of abundant extracellular matrix (ECM) and a relatively small number of chondrocytes embedded within the ECM. The chondrocytes produce the ECM, which consists mainly of collagens and proteoglycans. Various functions of chondrocytes are mediated through the cell-ECM interaction. Osteoarthritis is one of the most common joint diseases and is characterized by degeneration of the articular cartilage. Although the pathophysiology of osteoarthritis is not fully understood, the imbalance between breakdown and repair of the cartilage tissue is thought to be essential. ECM-degrading proteinases such as members of the matrix metalloproteinase (MMP) and a disintegrin and metalloproteinase with thrombospondin motifs (ADAMTS) gene families are known to play key roles in the breakdown of cartilage. ${ }^{1}$ On the other hand, chondrocyte cloning, also known as chondrocyte cluster formation, is frequently observed in osteoarthritic (OA) cartilage and represents a proliferative repair process that could reflect an effort by chondrocytes to repopulate the damaged cartilage tissue and enhance ECM synthesis. ${ }^{2-4}$ Despite the importance of chondrocyte cloning in cartilage repair, molecules involved and the mechanisms remain unclear.

Reversion-inducing cysteine-rich protein with Kazal motifs (RECK) is a membrane-anchored MMP regulator molecule. ${ }^{5,6}$ It is a glycosylphosphatidylinositol (GPI)-anchored protein and negatively regulates at least four MMPs (MMP-2, MMP-9, MT1-MMP, and MMP-7), ADAM10, and CD13 (aminopeptidase N). ${ }^{5-9}$ RECK is down-regulated in many cancer tissues, and restored expression of RECK in cancer cell lines results in reduced invasion, metastasis, and angiogenesis. ${ }^{5,6}$ RECK-deficient mice

Supported by the Ministry of Education, Culture, Sports, Science and Technology of Japan (grants-in-aid for Scientific Research (S) to Y.O. and for Young Scientists (B) to T.K.), the 21st Century Center of Excellence Program "Basic Study and Clinical Application of the Human Stem Cell Biology and Immunology" of Graduate School of Medicine, Keio University (to Y.O. and to T.K.) and Keio Gijuku Academic Development Funds (to T.K.).

Accepted for publication February 9, 2010

Supplemental material for this article can be found on http://ajp. amjpathol.org.

Address reprint requests to Yasunori Okada, M.D., Ph.D., Department of Pathology, School of Medicine, Keio University, 35 Shinanomachi, Shinjuku-ku, Tokyo 160-0016, Japan. E-mail: okada@sc.itc.keio.ac.jp. 
show embryonic lethality with severe disruption of mesenchymal and neural tissue integrity, suggesting its pivotal roles in tissue homeostasis. ${ }^{6}$ In contrast, tissue inhibitor of metalloproteinases (TIMP)-deficient animals are viable with only mild phenotypes. ${ }^{10-12}$ Although RECK is expressed in murine embryonic cartilage and may modulate chondrogenesis, ${ }^{13}$ little or no information is available for the expression and functions in adult human cartilage tissue and relevance to joint diseases.

In the present study, we examined the expression and localization of RECK in human articular cartilage tissues and found that RECK is up-regulated in OA cartilage, especially in cloning chondrocytes. We also analyzed regulation of RECK expression by cytokines and growth factors and the effects of RECK on spreading, migration, and proliferation using cultured OA chondrocytes.

\section{Materials and Methods}

\section{Clinical Samples and Histology}

Nonosteophytic articular cartilage samples were obtained at the time of arthroplasty from knee $(n=23)$ and hip $(n=4)$ joints of patients with osteoarthritis (mean \pm SD age, $72.3 \pm 10.3$ years) diagnosed according to the criteria of the American College of Rheumatology. ${ }^{14}$ Control normal cartilage samples were obtained from the hip joints $(n=9)$ of patients with femoral neck fracture (mean \pm SD age, $83.3 \pm 10.0$ years). The cartilage tissue samples were fixed with $4 \%$ paraformaldehyde, decalcified with 10\% EDTA ( $\mathrm{pH} 7.4$ ), and embedded in paraffin. Paraffin sections were stained with H\&E or toluidine blue and subjected to histological and histochemical grading described by Mankin et al. ${ }^{2}$ The degree of chondrocyte cloning was determined by morphometry according to our methods. ${ }^{15}$ Cartilage samples were also obtained from knee joints $(n=18)$ of patients with rheumatoid arthritis (RA) (mean \pm SD age, $58.9 \pm 9.4$ years) diagnosed according to the criteria of the American College of Rheumatology, ${ }^{16}$ and paraffin sections were made. Informed consent was obtained from the patients for the experimental use of the surgical samples according to the hospital ethics guidelines.

\section{Quantitative RT-PCR}

Total RNA was isolated directly from articular cartilage tissue (10 OA and 8 normal samples) and reverse-transcribed to cDNA as described previously. ${ }^{15}$ The cDNA was amplified in a TaqMan real-time PCR assay according to our methods. ${ }^{17,18}$ The relative quantification values of RECK were normalized by those of an endogenous control, glyceraldehyde-3-phosphate dehydrogenase $(\mathrm{GAPDH})$. For $\mathrm{GAPDH}$, the mixture of the PCR primers and probe was purchased from Applied Biosystems (Foster City, CA). Nucleotide sequences of the primers and probe for RECK were as follows: 5'-TGTTGACCTGTTTAGCGGATGT-3' (forward), 5'-GAAAAGTTCTGTTGGCCTGTTGT-3' (reverse), and 5'-FAM-CTTGCCAGTTGGGCTGTAGAAACCTTACTTACTGTA-TAMRA-3' (probe).
The total gene specificity of the sequences of these primers and probe and the absence of DNA polymorphisms were ascertained by BLASTN and Entrez (available at http://www.ncbi.n/m.nih.gov/).

\section{Immunohistochemistry}

Paraffin sections were pretreated by boiling in $10 \mathrm{mmol} / \mathrm{L}$ citrate buffer ( $\mathrm{pH}$ 6.0) for 10 minutes and subjected to immunohistochemistry for RECK and proliferating cell nuclear antigen (PCNA) using mouse monoclonal antiRECK antibody (5B11D12, $2.7 \mu \mathrm{g} / \mathrm{ml}),{ }^{5,6}$ rabbit polyclonal anti-PCNA antibody (FL-261, 1:200 dilution, Santa Cruz Biotechnology, Santa Cruz, CA), or nonimmune control IgG (BD Biosciences, Franklin Lakes, NJ). After the reaction with peroxidase-conjugated secondary antibodies (ENVISION+, Dako, Glostrup, Denmark), color was developed with diaminobenzidine and sections were counterstained with hematoxylin.

\section{OA Chondrocyte Cultures and Stimulation with Cytokines and Growth Factors}

Chondrocytes isolated from human OA cartilage by enzymatic dissociation were cultured in Dulbecco's modified Eagle's medium/Ham's F-12 (DMEM/F-12) (Sigma-Aldrich, St. Louis, MO) supplemented with 10\% fetal bovine serum (FBS) and $25 \mu \mathrm{g} / \mathrm{ml}$ ascorbic acid according to our methods. ${ }^{15}$ They were used for experiments at passage 1 to 2 , and a chondrocytic phenotype of the cultured OA cells was confirmed by the positive immunostaining of aggrecan and type II collagen according to our previous methods. ${ }^{19}$ Serumstarved OA chondrocytes were treated with insulin-like growth factor-1 (IGF-1) (Sigma-Aldrich), transforming growth factor- $\beta 1$ (TGF- $\beta 1$ ) (R\&D Systems, Minneapolis, $\mathrm{MN}$ ), interleukin (IL)-1 $\alpha$ (Dainippon, Osaka, Japan), tumor necrosis factor- $\alpha$ (TNF- $\alpha$ ) (Dainippon), hepatocyte growth factor (HGF) (R\&D Systems), basic fibroblast growth factor (bFGF) (Sigma-Aldrich), or vascular endothelial growth factor $\left(V G_{165}\right)$ (R\&D Systems) in serum-free DMEM/F-12 containing $0.2 \%$ lactalbumin hydrolysate for 24 hours (for RT-PCR analysis) or 48 hours (for immunoblotting).

\section{$R T-P C R$}

Total RNA extracted from OA chondrocytes was subjected to RT-PCR as described previously. ${ }^{15}$ The PCR primers used were as follows: for RECK, 5'-CCTCAGTGAGCACAGTTCAGA-3' (forward) and 5'-GCAGCACACACACTGCTGTA-3' (reverse) 20 ; and for GAPDH, 5'CCACCCATGGCAAATTCCATGGCA-3' (forward) and 5'-TCTAGACGGCAGGTCAGGTCCACC-3' (reverse). ${ }^{15}$ Expected sizes of the PCR products of RECK and GAPDH were 477 and $600 \mathrm{bp}$, respectively. 


\section{Immunoblotting}

Cultured OA chondrocytes were washed twice with icecold PBS and lysed in $25 \mathrm{mmol} / \mathrm{L}$ Tris- $\mathrm{HCl}, \mathrm{pH} 7.5,150$ $\mathrm{mmol} / \mathrm{L} \mathrm{NaCl}, 1 \%$ Triton X-100, 0.1\% SDS, and Protease Inhibitor Cocktail Complete Mini (Roche, Basel, Switzerland) (the lysis buffer). Total cell lysates were subjected to SDS-polyacrylamide gel electrophoresis under reducing conditions. The amount of loaded samples was adjusted to equal cell number using a PicoGreen doublestranded DNA quantitation kit (Molecular Probes, Eugene, OR). For analysis of tissue extracts of OA cartilage, cartilage tissue was minced, frozen with liquid nitrogen, and pulverized in a Cool Mill (Toyobo, Osaka, Japan). The powdered tissue was lysed in $50 \mathrm{mmol} / \mathrm{L}$ Tris- $\mathrm{HCl}$, $\mathrm{pH} 8.0,8 \mathrm{~mol} / \mathrm{L}$ urea, $1 \mathrm{mmol} / \mathrm{L}$ dithiothreitol, and 1 $\mathrm{mmol} / \mathrm{L}$ EDTA at $4^{\circ} \mathrm{C}$ for 2 hours. Immunoblotting was performed using specific antibodies as follows: antiRECK (5B11D12), anti-GAPDH (Abcam, Cambridge, MA), anti-focal adhesion kinase (FAK) (Santa Cruz Biotechnology), anti-phospho-FAK (Tyr ${ }^{925}$ ) (Cell Signaling Technology, Danvers, MA), anti-phospho-FAK (Tyr ${ }^{576 / 577}$ ) (Cell Signaling Technology), anti-phospho-FAK (Tyr ${ }^{397}$ ) (BD Biosciences), anti-extracellular signal-regulated kinase (ERK) 1/2 (Cell Signaling Technology), and anti-phosphoERK1/2 (Cell Signaling Technology).

\section{Treatment of Chondrocytes with Phosphatidylinositol-Specific Phospholipase C}

OA chondrocytes were incubated with PBS or phosphatidylinositol-specific phospholipase C (PI-PLC) $(1 \mathrm{U} / \mathrm{ml}$ in PBS, Molecular Probes) on ice for 30 minutes and fixed with ethanol. Cells were incubated with anti-RECK antibody and then with Alexa Fluor 488-conjugated secondary antibody (Molecular Probes). Nuclei were stained with TO-PRO-3 (Molecular Probes). PBS and PI-PLC solution used above (supernatant) were collected and concentrated by Amicon Ultra (Millipore, Billerica, MA). Lysates of treated cells were also collected. The samples were subjected to SDS-PAGE and immunoblotting for RECK.

\section{Transfection of Small Interfering RNA or Expression Plasmids}

Small interfering RNAs (siRNAs) designed to target RECK, ie, 5'-AAGACCCAGCCCUUGCCUCAA-3' (siRNA1) ${ }^{21}$ and 5'-GGUGUGUUUAAGAAGUCUGTT-3' (siRNA2) (siRNA 29149, Ambion, Austin, TX), and a nonsilencing control RNA, 5'-AACGUUGCGAUAGCGUAGUAC-3', 21 were synthesized. The expression vector containing human RECK cDNA (pCXN2-hRECK) and empty vector (pCXN2) were prepared. ${ }^{6}$ Transfection of OA chondrocytes was performed by electroporation using a Nucleofector kit (Amaxa, Gaithersburg, MD) according to the manufacturer's protocols. Cultured OA chondrocytes (passage 0 to 1) were removed from a dish by enzymatic digestion with collagenase (Roche) and pronase (Calbiochem, Darmstadt, Germany), divided into three groups, and transfected with nonsilencing RNA, siRNA1, or siRNA2. A similar method was used for transfection of the RECK expression vector and empty vector. Thus, the transfected chondrocytes used in the comparative studies were always at the same passage number. Cotransfection of the green fluorescent protein (GFP) expression vector (pmaxGFP, Amaxa) was done to monitor successfully transfected cells.

\section{Cell Adhesion and Spreading Assays}

Adhesion of siRNA-transfected OA chondrocytes to plates was assayed according to previous methods 22,23 with some modifications. In brief, chondrocytes removed from culture dishes with $0.02 \%$ EDTA in PBS were washed and resuspended in DMEM/F-12. Equal numbers of cells were added to 96-well plates, which had been coated with or without fibronectin (BD Biosciences) or type II collagen (Koken, Tokyo, Japan) and allowed to adhere at $37^{\circ} \mathrm{C}$ for 1 hour. The wells were washed briefly with PBS, and adherent cells were fixed in $20 \%$ formalin and stained with $1 \%$ methylene blue. The dye was solubilized in a $1: 1$ mixture of ethanol and $0.1 \mathrm{~N} \mathrm{HCl}$, and absorbance at $650 \mathrm{~nm}$ was measured. For evaluation of cell spreading, ${ }^{24}$ equal numbers of dissociated chondrocytes were plated on 12-well plates (Nunclon Delta Surface, Nunc, Roskilde, Denmark) cultured at $37^{\circ} \mathrm{C}$ for 1 to 3 hours in the presence or absence of $10 \mu \mathrm{mol} / \mathrm{L}$ BB94 (British Biotech, Oxford, UK) and then were stained with $1 \%$ methylene blue after fixation with $4 \%$ paraformaldehyde. The cell surface area on the plates was measured using Scion Image software (Scion, Frederick, MD) for more than 80 cells in each condition.

\section{Fluorescent Staining of Chondrocytes Cultured in Monolayer or in Three-Dimensional Collagen $\mathrm{Gel}$}

For cell morphology analysis, culture dishes (Nunclon Delta Surface) were noncoated or coated with fibronectin or type II collagen. OA chondrocytes were cultured on these dishes for 48 hours in the presence or absence of $10 \mu \mathrm{mol} / \mathrm{L}$ BB94, $20 \mu \mathrm{mol} / \mathrm{L}$ GM6001 (Calbiochem), or $0.1 \mu \mathrm{mol} / \mathrm{L}$ TIMP-2 (R\&D Systems), fixed with $4 \%$ paraformaldehyde for 15 minutes, and permeabilized with $0.2 \%$ Triton $X-100$ in PBS for 10 minutes. Cells were incubated with anti-paxillin (BD Biosciences) or anti-vinculin (Sigma-Aldrich) antibody and then with Alexa Fluor 594-conjugated secondary antibody (Molecular Probes). F-actin was visualized with Texas Red-conjugated phalloidin (Molecular Probes). Nuclei were stained with TOPRO-3. Stained cells were imaged on a confocal microscope (FV300, Olympus, Tokyo, Japan). Transfected OA chondrocytes were also cultured at a cell density of $7 \times$ $10^{5}$ cells $/ \mathrm{ml}$ in $0.4 \%$ atelocollagen gel (Koken) for 48 hours or 10 days as described previously, ${ }^{25}$ and F-actin and nuclei were imaged with Texas Red-conjugated phalloidin and TO-PRO-3, respectively. 
A

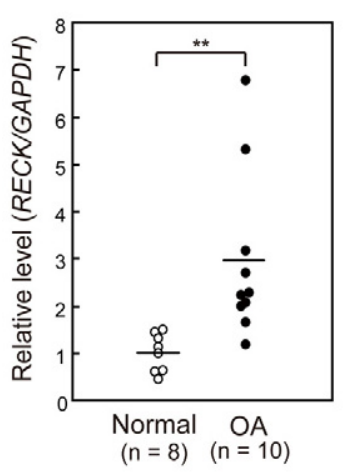

C

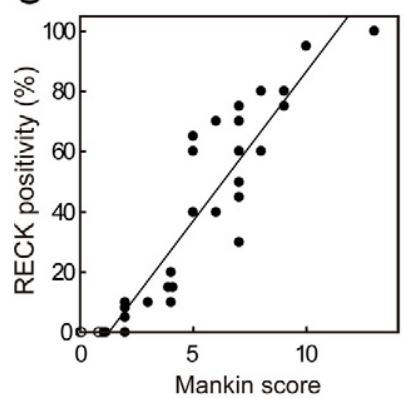

B

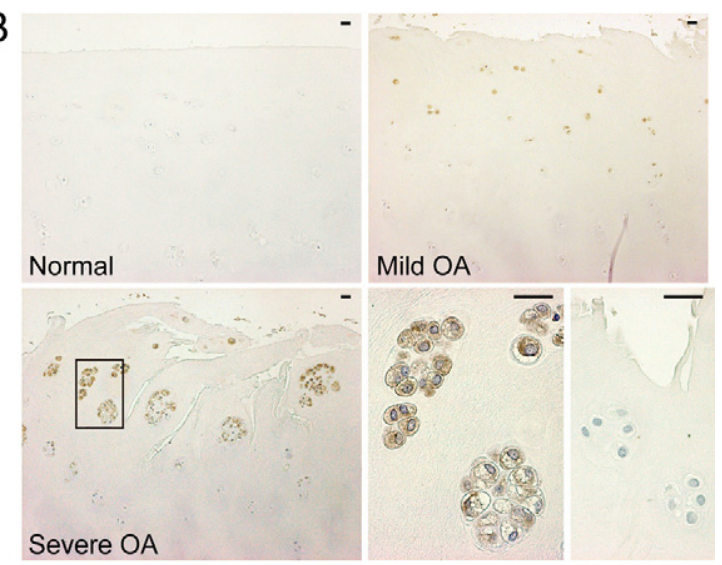

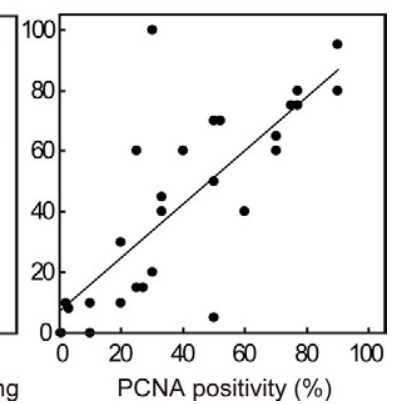

Figure 1. Enhanced expression of RECK in human OA cartilage and correlation between RECK immunoreactivity and cartilage parameters. A: Quantitative analysis of RECK gene expression in normal and OA cartilage tissue samples. Relative RECK/GAPDH mRNA levels were measured by quantitative RT-PCR. Horizontal bars indicate mean values. ${ }^{* *} P<0.01$. B: Immunohistochemical analysis of RECK in normal and OA cartilage samples. Paraffin sections of normal cartilage (Mankin score 1), mild OA cartilage (Mankin score 4), and severe OA cartilage (Mankin score 8) were immunostained with antiRECK antibody. The lower center panel is the high-power view of the boxed area in the lower left panel. The lower right panel shows severe OA cartilage immunostained with nonimmune IgG. Scale bars $=25 \mu \mathrm{m}$. C: Correlations of RECK immunoreactivity with Mankin score (left), degree of chondrocyte cloning (center), and PCNA positivity (right). Open circle, normal cartilage samples $(n=9)$; Closed circle, OA cartilage samples $(n=27)$.

\section{Monolayer Wounding Assay}

Cell migration activity was evaluated by a monolayer wounding assay. ${ }^{26}$ Transfected OA chondrocytes were grown to confluence on plastic culture plates and then were cultured in DMEM/F-12 containing $10 \%$ FBS and 5 $\mathrm{mmol} / \mathrm{L}$ hydroxyurea to inhibit cell proliferation. ${ }^{27} \mathrm{~A}$ uniform straight wound was made in the cell monolayer using a 200- $\mu$ l pipet tip. After floating cells were washed out, the cells were cultured at $37^{\circ} \mathrm{C}$ for indicated times. The open wound area was measured on photographs of wounds using Scion Image software, and the wound closure rate was calculated.

\section{5-Bromo-2'-Deoxyuridine Incorporation Assay}

Transfected OA chondrocytes were serum-starved overnight and cultured with DMEM/F-12 containing $10 \mu \mathrm{mol} / \mathrm{L}$ 5-bromo-2'-deoxyuridine (BrdU) in the presence or absence of 1 to $5 \%$ FBS (for 12 hours) or $300 \mathrm{ng} / \mathrm{ml} \mathrm{IGF-1}$ and/or $10 \mathrm{ng} / \mathrm{ml}$ TGF- $\beta 1$ (for 45 hours). The cells were fixed with ethanol, treated with $2 \mathrm{~N} \mathrm{HCl}$ at $37^{\circ} \mathrm{C}$ for 60 minutes, and then immunostained with anti-BrdU antibody (Novocastra, Newcastle on Tyne, UK). BrdU-positive nuclei/total nuclei were counted under a microscope.

\section{Statistical Analysis}

A Mann-Whitney U-test was used to compare the data from $O A$ and normal cartilage samples. For the data from experiments using OA chondrocytes, statistical signifi- cance was determined by a Student's t-test. $P<0.05$ was considered significant.

\section{Results}

\section{Expression of RECK mRNA in Articular Cartilage}

The relative mRNA expression level of RECK (RECK/ GAPDH ratio) was measured by quantitative RT-PCR. As shown in Figure 1A, it was significantly higher in $\mathrm{OA}$ cartilage (mean $\pm \mathrm{SD}, 2.93 \pm 1.76$ ) than in normal control cartilage (mean $\pm \mathrm{SD}, 1.00 \pm 0.41)(P<0.01)$.

\section{Immunolocalization of RECK in Articular Cartilage}

Immunohistochemical staining of articular cartilage tissue demonstrated that RECK was localized to the cytoplasm and cell membranes of the chondrocytes in OA samples, whereas negligible staining was present in normal samples (Figure 1B). In the mild to moderate OA cartilage tissue, RECK signals were restricted to chondrocytes near the erosive surface. Chondrocytes in the deeper zone showed negligible immunoreactivity. In the severe OA cartilage tissue, RECK expression was detected in many chondrocytes, especially in cloning (clustering) chondrocytes, whereas chondrocytes without cloning located in the deeper zone were negative for RECK (Figure 1B). When RECK immunoreactivity (the percentage of immunostained chondrocytes to total cells) was plotted 
A

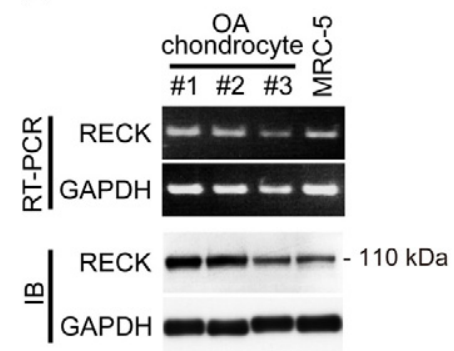

C

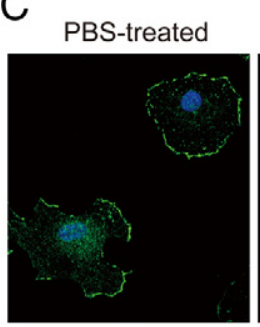

B

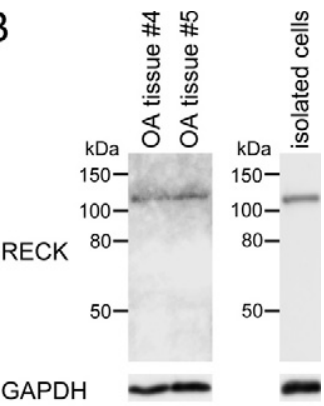

D

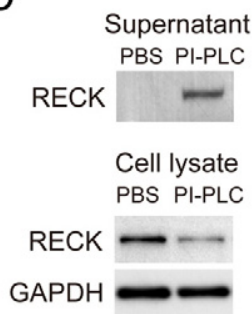

Figure 2. Expression of RECK in cultured OA chondrocytes. A: Expression of RECK in the chondrocytes from three different patients (\#1 to \#3). mRNA and protein expression of RECK were examined by RT-PCR and immunoblotting (IB). The MRC-5 cell line is used as positive control. ${ }^{5}$ B: Immunoblotting of RECK in the tissue extracts of OA cartilage from two patients (\#4 and $\# 5$ ) and cell lysates of freshly isolated OA chondrocytes. Molecular size markers are indicated. C: Immunostaining of RECK (green) in OA chondrocytes treated with PBS or PI-PLC (1 U/ml in PBS). Nuclei are stained with TO-PRO-3 (blue). Scale bar $=50 \mu \mathrm{m}$. D: Immunoblotting of RECK in supernatants and cell lysates of PBS- or PI-PLC-treated chondrocytes.

against Mankin score (histological severity of OA), a strong linear correlation was observed $(r=0.93, P<$ $0.0001, n=36$ ) (Figure 1C). RECK immunoreactivity was also correlated with the degree of chondrocyte cloning $(r=0.73, P<0.0001, n=36)$ and PCNA-positive cell index $(r=0.85, P<0.0001, n=36)$ (Figure 1C).

We also immunolocalized RECK in RA cartilage and compared the immunoreactivity between $\mathrm{OA}$ and RA samples. RECK was sporadically immunostained in the chondrocytes in RA cartilage (Supplemental Figure 1A, see http://ajp.amjpathol.org). However, the RECK immunoreactivity was much lower in RA $(6.1 \pm 12.8 \%)$ than in OA samples (44.0 $\pm 31.3 \% ; P<0.01$ ) (Supplemental Figure 1B, see http://ajp.amjpathol.org).

\section{RECK Expression on the Cell Surface of Cultured OA Chondrocytes via GPI Anchoring}

When RECK expression was examined in cultured OA chondrocytes by RT-PCR and immunoblotting, both RECK mRNA and protein of $110 \mathrm{kDa}$ were detected (Figure 2A). Immunoblotting analysis of tissue extracts of OA cartilage and cell lysates of freshly isolated OA chondrocytes also showed the RECK protein expression (Figure 2B). Immunofluorescent microscopy showed strong RECK signals on the cell membranes in addition to weak staining in the cytoplasm, and the immunostaining on the cell membranes was reduced in the chondrocytes treated with PI-PLC (Figure 2C). In accord with this finding, the RECK protein was shifted from the cell fraction to the supernatants by the PI-PLC treatment (Figure 2D).

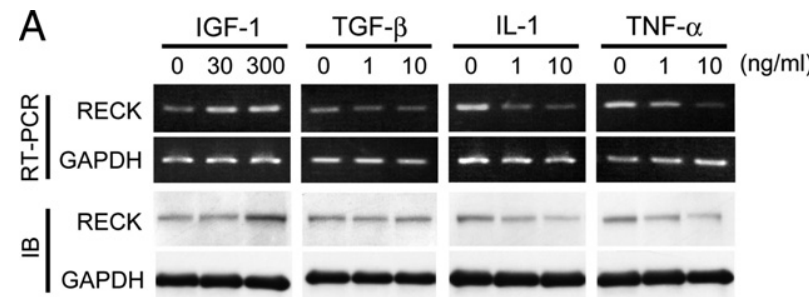

B

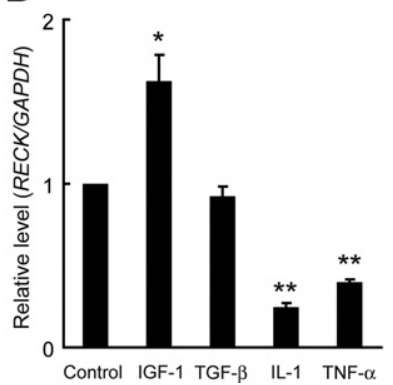

C

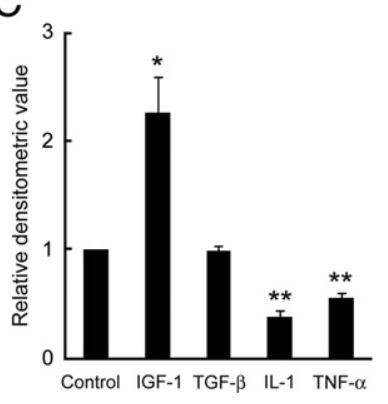

Figure 3. Effects of cytokines and growth factors on RECK expression in OA chondrocytes. A: Effects of IGF-1, TGF- $\beta 1$, IL- $1 \alpha$, and TNF- $\alpha$ on RECK expression in OA chondrocytes. RECK expression was examined by RT-PCR and immunoblotting (IB). B and C: Quantitative RT-PCR analysis (B) and densitometric quantification of RECK signals on immunoblot (C). Concentrations of IGF- 1 , TGF- $\beta 1$, IL- $1 \alpha$, and TNF- $\alpha$ are $300,10,10$, and $10 \mathrm{ng} / \mathrm{ml}$, respectively. Values are means and SEM of three independent experiments. ${ }^{*} P<0.05 ;{ }^{* *} P<0.01$ versus control.

These results indicate that RECK protein is expressed on the cell surface of cultured OA chondrocytes by GPI anchoring.

\section{Effects of Cytokines and Growth Factors on RECK Expression in OA Chondrocytes}

Serum-starved OA chondrocytes were treated with IGF-1, TGF- $\beta 1$, IL- $1 \alpha$, TNF- $\alpha$, HGF, bFGF, or VEGF VI65 $_{1}$, and RECK expression was determined by RT-PCR and immunoblotting. Among these factors, the expression of RECK mRNA and protein seemed to be up-regulated by IGF-1 and down-regulated by IL- $1 \alpha$ and TNF- $\alpha$ (Figure 3A). TGF- $\beta 1$, HGF, bFGF, and VEGF ${ }_{165}$ had no significant effects (Figure $3 \mathrm{~A}$ for TGF- $\beta 1$ and data not shown for others). Quantitative RT-PCR analysis and densitometric analysis of RECK protein bands confirmed these findings (Figure 3, B and C).

\section{Effects of RECK on Chondrocyte Adhesion, Spreading, Morphology, and Focal Adhesions}

When OA chondrocytes were transfected with two different siRNAs targeting RECK ( silencing control RNA (NS), expressions of RECK mRNA and protein were effectively suppressed by siRNA1 or siRNA2 to approximately $10 \%$ of the control levels (Figure $4 A$ ). Because RECK protein was localized mainly on the cell surface, we explored changes in the cell-matrix interaction of OA chondrocytes caused by RECK silencing. In the cell adhesion assay, there was no difference in the chondrocyte attachment to ECM (fibronectin or type II 
A
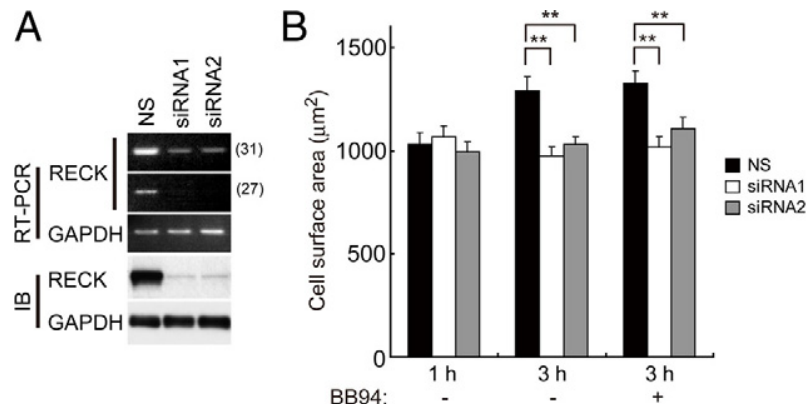

C

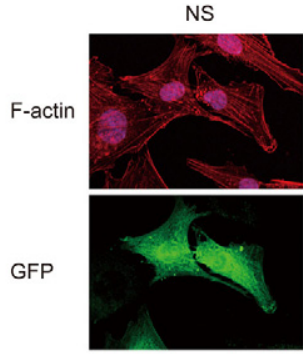

SiRNA1
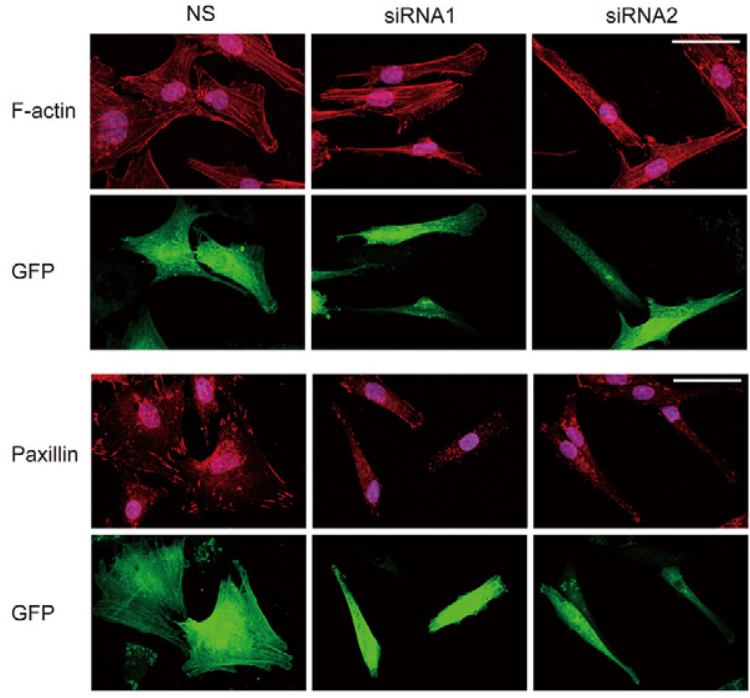

GFP

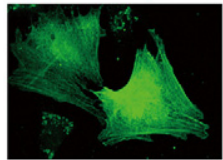

NS

D
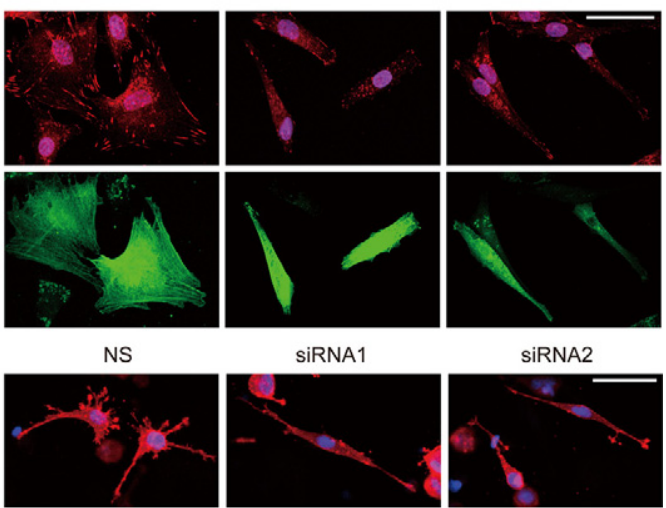

$48 \mathrm{~h}$
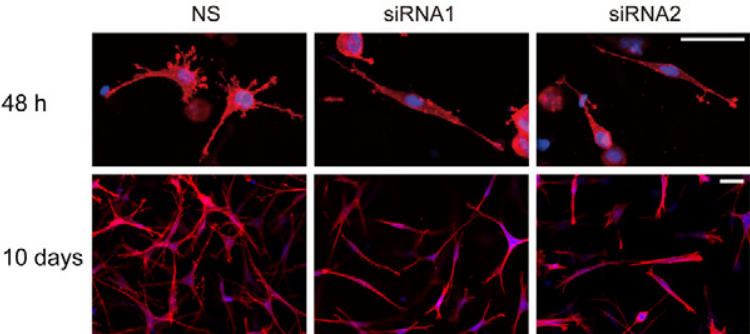

Figure 4. Effects of RECK on cell spreading, morphology, and focal adhesions of OA chondrocytes and on morphology of the cells in three-dimensional culture. A: Down-regulation of RECK with two different siRNAs targeting RECK (siRNA1 and siRNA2). The RECK expression was examined by RT-PCR at 27 or 31 cycles and immunoblotting (IB). NS, nonsilencing control. B: Effect of RECK on cell spreading of OA chondrocytes. Cell spreading was evaluated 1 hour and 3 hours after plating on plastic culture plates in the presence or absence of BB94 $(10 \mu \mathrm{mol} / \mathrm{L})$. Cell surface areas of more than 80 cells were measured in each condition. Bars, mean and SEM. ${ }^{* *} P<0.01$. C: Morphology and focal adhesions of transfected OA chondrocytes. F-actin visualized by Texas Red-phalloidin and focal adhesions immunostained with anti-paxillin antibody (red) are shown in upper and lower panels, respectively. Nuclei are stained with TO-PRO-3 (blue). Fluorescence (green) of cotransfected GFP of the same cells is also shown. Scale bars $=50 \mu \mathrm{m}$. D: Effects of RECK on morphology of OA chondrocytes in three-dimensional culture. They were cultured for 48 hours or 10 days. Texas Red-phalloidin staining for F-actin (red) and nuclear staining with TO-PRO-3 (blue). Scale bars $=50 \mu \mathrm{m}$

collagen)-coated or noncoated plates between NS- and siRNA-transfected cells (data not shown). However, cell spreading on noncoated plates or ECM-coated plates was significantly suppressed in siRNA-transfected cells compared with NS-transfected cells at 3 hours after plating (Figure 4B for noncoated plates and data not shown for ECM-coated plates). Similar results were obtained in the presence of BB94 (a broad spectrum synthetic MMP inhibitor) (Figure 4B), indicating that this effect of RECK is independent of its MMP-inhibiting activity.

Steady-state cell morphology of the transfected OA chondrocytes labeled by GFP was compared at 48 hours after plating on noncoated surfaces. NS-transfected cells showed polygonal shape with actin stress fibers of multiple directions, but siRNA-transfected cells demonstrated bipolar shape with parallel running of actin fibers (Figure 4C, upper panel). When these cells were immunostained with antibodies against paxillin or vinculin (marker proteins for focal adhesions), focal adhesions of NS-transfected cells showed large and long shape, but those of siRNA-transfected cells showed small, dot-like morphology (Figure 4C, lower panel for paxillin and data not shown for vinculin immunostaining). Very similar results were obtained with OA chondrocytes cultured on ECM (fibronectin or type II collagen)-coated surfaces (data not shown). We further examined the possible involvement of MMP inhibitor activity of RECK in the morphological changes by performing the experiments in the presence of BB94, GM6001, or TIMP-2, all of which are MMP inhibitors. The results showed that there is no difference in the findings in the presence or absence of BB94, GM6001, or TIMP-2 (Supplemental Figure 1C, see http://ajp.amjpathol.org), indicating that this is independent of the inhibitor activity.

When the transfected OA chondrocytes were cultured for 48 hours in three-dimensional collagen gel, NS-transfected cells evolved to a stellate shape with multiple cell processes, but siRNA-transfected cells demonstrated a bipolar shape with few cell processes (Figure 4D). These morphological characteristics of NS- or siRNA-transfected chondrocytes were maintained or even exaggerated at 10 days after the culture, and the cellularity in the collagen gel was increased in NS-transfected cells compared with siRNA-transfected cells (Figure 4D).

\section{Effects of RECK on Chondrocyte Migration}

The morphological data on the two- or three-dimensional cultures of chondrocytes suggested the possibility that RECK suppresses chondrocyte migration by modulation of focal adhesion and cytoskeleton, both of which are the key structures influencing cell migration. ${ }^{28}$ Therefore, we evaluated effects of RECK on the chondrocyte migration by monolayer wounding assay. As shown in Figure 5, A and $B$, siRNA-transfected OA chondrocytes migrated significantly faster than NS-transfected cells. BB94 significantly inhibited the promoted migration of siRNAtransfected cells, but little effect on NS-transfected cells was observed (Figure 5C), suggesting that the suppression of chondrocyte migration by RECK protein is ascribed, at least in part, to MMP inhibition by RECK. On the other hand, when RECK-overexpressing chondrocytes were prepared using the expression vector of RECK, their migration was significantly inhibited compared with empty vector-transfected controls (Figure 5D). 
A

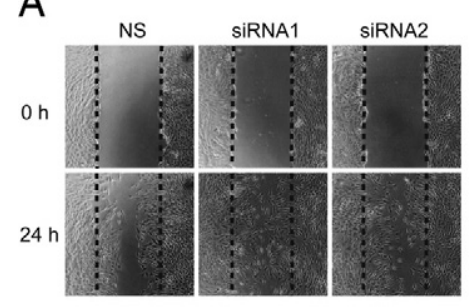

B

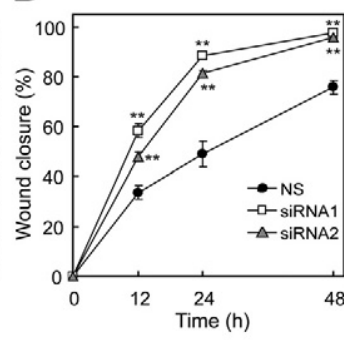

C

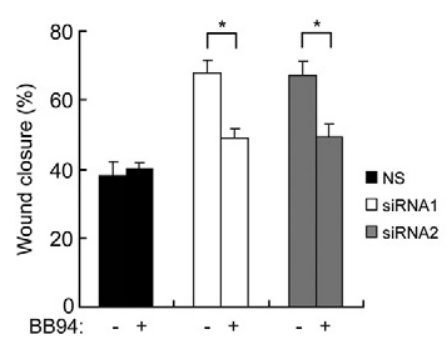

Figure 5. Effects of RECK on migration of OA chondrocytes. A: Representative micrographs of monolayer wounding assay. Straight wounds (between dashed lines) were made at 0 hours, and migrated chondrocytes were monitored. Representative photographs at 24 hours after wounding are shown. B: Wound closure rate of chondrocytes transfected with siRNAs at indicated times after wounding. Bars, mean \pm SEM. ${ }^{* *} P<0.01$ versus NS at the same time point. C: Wound closure rates at 24 hours after wounding in the presence of BB94 $(10 \mu \mathrm{mol} / \mathrm{L})$ or DMSO (vehicle). Bars, mean and SEM ${ }^{*} P<0.05$. D: Suppressed wound closure rate by overexpression of RECK. Chondrocytes were transfected with control empty vector (C) or RECK expression vector (R). ${ }^{*} P<0.05$; ${ }^{* * *} P<0.01$. Inset: Validation of RECK overexpression by immunoblotting.

\section{Effects of RECK on Chondrocyte Proliferation}

FBS, IGF- 1 , and TGF- $\beta 1$ are known to stimulate chondrocyte proliferation. ${ }^{29,30}$ Thus, we examined the effects of RECK on the proliferation of OA chondrocytes treated with these factors by a BrdU incorporation assay. As shown in Figure 6, A and B, the treatment with FBS, TGF- $\beta 1$ alone, or IGF- $1+$ TGF- $\beta 1$ promoted proliferation, and the IGF- 1 treatment tended to stimulate proliferation although it was not statistically significant. Down-regulation of RECK by siRNA significantly reduced the FBS- or growth factor-stimulated proliferation (Figure 6, A and B). This effect was not changed by addition of BB94, GM6001, or TIMP-2 (Figure 6, A and B, Supplemental Figure 1D, see http://ajp.amjpathol.org). In addition, overexpression of RECK enhanced proliferation in the chondrocytes only under stimulation with FBS or IGF- $1+$ TGF- $\beta 1$ (Figure 6, C and D). These data indicate that RECK has a positive role in OA chondrocyte proliferation, although MMP inhibition by this molecule is not involved directly in this process.

\section{Effects of RECK on Cellular Signaling Events}

To explore a possible link between changes in focal adhesions and cell proliferation affected by RECK, we examined the influence of RECK down-regulation on FAK and ERK signaling in FBS-stimulated chondrocytes, because FAK is one of the most important signaling molecules associated with focal adhesions, ${ }^{31}$ and ERK is one of the major down-

A

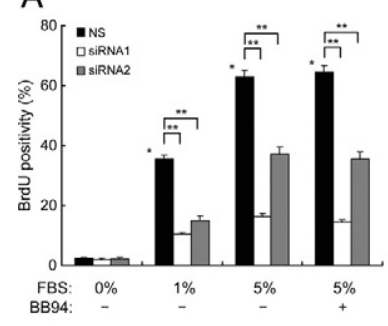

B
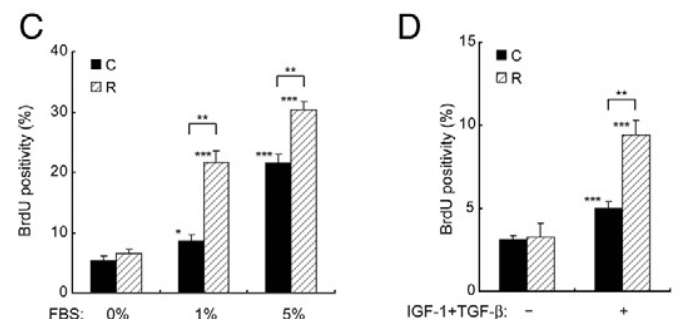

Figure 6. Effects of RECK on proliferation of OA chondrocytes induced with serum or IGF-1+TGF- $\beta 1$. A and B: Down-regulation of chondrocyte proliferation by siRNAs targeting RECK. NS- or siRNA-transfected chondrocytes were stimulated with FBS (A) or IGF-1, TGF- $\beta$, or IGF-1+TGF- $\beta$ (B) in the presence or absence of $10 \mu \mathrm{mol} / \mathrm{L} \mathrm{BB94}$, and BrdU incorporation was measured. Bars, mean and SEM. ${ }^{* * *} P<0.01 ;{ }^{*} P<0.01$ versus NS without mitogen. $\mathbf{C}$ and $\mathbf{D}$ : Enhanced chondrocyte proliferation by overexpression of RECK. Chondrocytes transfected with control empty vector (C) or RECK expression vector (R) were stimulated with FBS (C) or IGF-1+TGF- $\beta$ (D) ${ }^{*}{ }^{*} P<0.01 ;{ }^{*} P<0.05 ;{ }^{*}{ }^{*}{ }^{*} P<0.01$ versus the same transfectant without mitogen.

stream effectors of FAK signaling relevant to proliferation. ${ }^{32}$ As shown in Figure 7, major phosphorylation sites of FAK $\left(\mathrm{Tyr}^{925}, \mathrm{Tyr}^{576 / 577}\right.$, and $\mathrm{Tyr}^{397}$ ) were less phosphorylated in siRNA-transfected chondrocytes than in NS-transfected cells. Phosphorylation of ERK was also suppressed in RECK-down-regulated chondrocytes.

\section{Discussion}

In the present study, we have provided the first evidence that RECK is up-regulated by chondrocytes in articular cartilage of human OA. Quantitative real-time PCR shows that

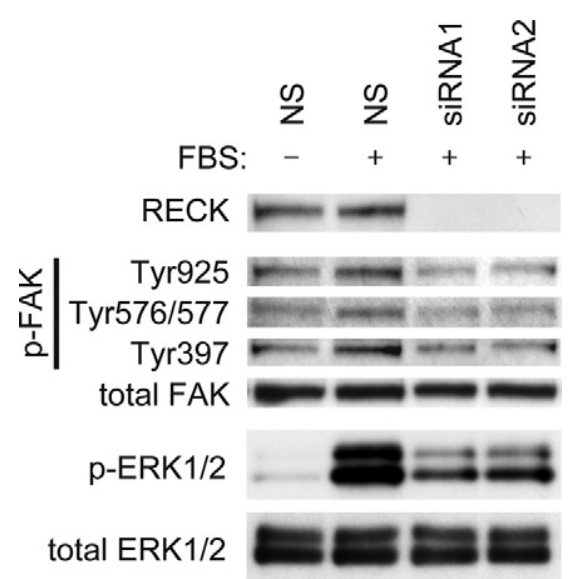

Figure 7. Effects of RECK on FAK and ERK signaling in OA chondrocytes Transfected chondrocytes were serum-starved and stimulated with $10 \%$ FBS for 10 minutes, and then cell lysates were analyzed by immunoblotting using antibodies against RECK, FAK, phosphorylation (p)-specific FAK (Tyr ${ }^{225}$, $\mathrm{Tyr}^{576 / 577}$, and $\mathrm{Tyr}^{397}$ ), ERK1/2, and phosphorylation-specific ERK1/2. 
the mRNA expression level of RECK is 3-fold higher in OA cartilage than in control normal cartilage. Immunolocalization with specific antibody also shows that chondrocytes located in the affected zones of OA cartilage express RECK protein and the positivity levels of immunostaining increase with severity of OA changes. In mouse embryos, RECK is widely expressed in primordial tissues for various organs such as blood vessels, ${ }^{6}$ skeletal muscle, ${ }^{33}$ lung, ${ }^{34}$ brain, ${ }^{8}$ and cartilage. ${ }^{13}$ Although little or no information is available about the RECK expression by chondrocytes in adult mice, the expression by chondrocytes during cartilage development is known in embryonic day 13.5 to 16.5 mouse embryos, and the expression becomes up-regulated in the later phase of chondrocytic differentiation of a chondrogenic mouse cell line ATDC5. ${ }^{13}$ Clinical relevance of RECK has been reported in many types of cancers and a small number of chronic inflammatory diseases. In malignant tumors, RECK is generally down-regulated, and a higher level of residual RECK expression correlates with better prognosis in various solid tumors. ${ }^{35}$ Synovial tissues from patients with rheumatoid arthritis, osteoarthritis, or trauma are reported to express RECK, and the expression level is the lowest in rheumatoid arthritis. ${ }^{36}$ Our findings in the present study, however, add human OA cartilage in a list of tissues with RECK overexpression in non-neoplastic diseases.

Up-regulation of RECK in OA cartilage suggests the possibility that the expression by chondrocytes is regulated by growth factors and/or proinflammatory cytokines that are commonly present in $\mathrm{OA}$ joint tissues. This prompted us to study the effects of seven factors (IGF-1, TGF- $\beta 1, \mathrm{IL}-1, \mathrm{TNF}-\alpha, \mathrm{HGF}, \mathrm{bFGF}$, and VEGF) on RECK gene expression, and we found that the expression is promoted by IGF-1 and down-regulated by IL-1 and TNF- $\alpha$. Several suppressors of the RECK gene expression have been reported. Transfection of oncogenes such as Ha-ras, HER-2/neu, or Epstein-Barr virus latent membrane protein 1 to NIH3T3 cells or carcinoma cells represses the RECK gene expression through binding of $\mathrm{Sp} 1$ and Sp3 proteins to the binding site in the promoter. ${ }^{20,37,38}$ MicroRNA 21 is known to play a key role in down-regulation of the RECK expression in human glioma cells and gastric cancer cells, ${ }^{39,40}$ and granulocytecolony stimulating factor induces suppression of the expression in human and murine hematopoietic cells in the phosphatidylinositol 3-kinase/Akt-dependent manner. ${ }^{41}$ On the other hand, only TIMP-2 is known to promote the RECK gene expression in human microvascular endothelial cells through the $\alpha 3 \beta 1$ integrin-mediated activation of Rap 1. ${ }^{42,43}$ The present study is the first to demonstrate that IGF-1, IL-1, and TNF- $\alpha$ can be involved in the regulation of the RECK expression in OA chondrocytes, although the intracellular signaling pathways for the regulation of the gene expression remain to be elucidated by further work. Previous in situ hybridization and immunohistochemical studies on OA cartilage have shown that the expression of IGF- 1 is observed mainly in the superficial and cloning chondrocytes, whereas IL-1 and TNF- $\alpha$ are expressed predominantly in chondrocytes in the intermediate and deep zones. ${ }^{44,45}$ The different expression pattern of these factors seems to be consistent with the RECK expression observed mainly in the cloning chon- drocytes in OA cartilage. Our study also demonstrated that the RECK immunoreactivity is significantly lower in RA cartilage than in OA cartilage. Because the levels of $\mathrm{IL}-1$ and TNF- $\alpha$ in synovial fluids are much higher in rheumatoid arthritis than in osteoarthritis, ${ }^{46}$ it is interesting to speculate that the decrease in number of chondrocytes in RA cartilage may be ascribed to the downregulation of RECK expression by these cytokines.

RECK was originally discovered as a cDNA clone inducing flat reversion in $\mathrm{NIH} 3 \mathrm{~T} 3$ cells transformed by the $\mathrm{v}$-K-ras oncogene and shown to inhibit both secretion and activity of MMP-9. ${ }^{5}$ Subsequent studies have demonstrated that RECK negatively regulates several members of the MMP gene family including MMP-2, MT1MMP, ${ }^{6}$ and MMP- $7,{ }^{9}$ and other metalloproteinases such as $\mathrm{ADAM} 10^{8}$ and $\mathrm{CD} 13$ (aminopeptidase N). ${ }^{7}$ Although these studies indicate that RECK acts as a metalloproteinase inhibitor, RECK differs in several points from TIMPs, which are general MMP inhibitors. First, RECK deficiency in mice is embryonic lethal, whereas knockout mice of the TIMP species exhibit only minor phenotypes. ${ }^{6,10-12}$ Second, RECK, but not TIMPs, can modulate endocytic pathways of MT1-MMP and CD13, and this function is closely related to lipid rafts (detergent-resistant membrane). ${ }^{7}$ Third, as shown in the present and previous studies, ${ }^{5,47}$ RECK is expressed on the cell membranes via GPI anchoring. From these functional variety and membrane localization of RECK, it may be possible to think that RECK has non-MMP inhibitor functions such as a modulator for the cell-ECM interaction of chondrocytes. In our study, we have shown that down-regulation of RECK in cultured OA chondrocytes induces decreased cell spreading and smaller focal adhesions together with morphological changes from polygonal to bipolar shape. These alterations by RECK silencing seem to be due to non-MMP inhibitor activity of RECK, because these effects were not influenced by addition of MMP inhibitors (BB94, GM6001, and TIMP-2) or by ECM coating of the plates. Cell spreading and maintenance of cell shape require the interaction between integrin-mediated adhesion structures and the surface of plates, ${ }^{48,49}$ and chondrocytes in culture and cartilage tissue are known to possess paxillin/vinculin-positive focal adhesions. ${ }^{49,50}$ In fact, our study showed that focal adhesions are well organized in cultured OA chondrocytes and seem to be disorganized by the RECK down-regulation. A recent study using mouse embryonic fibroblasts indicated that RECK could stabilize focal adhesions and anteroposterior polarity of the cells. ${ }^{47}$ Although the mechanisms by which RECK modulates cell spreading and morphology of OA chondrocytes remain elusive, it seems likely that RECK plays a role in the regulation of focal adhesions in OA chondrocytes via its non-MMP inhibitor activity.

Migration activity of chondrocytes within cartilage tissue is believed to be minimal. ${ }^{51}$ However, chondrocytes in cartilage tissue are endowed with morphological features of "motile cells," which include abundant filopodias, centrioles, cilia, and cytoskeletal fibers. ${ }^{52,53}$ In addition, isolated chondrocytes show migration under culture conditions on planar surfaces or even within three-dimensional matrices. ${ }^{51,54}$ In the present study, we confirmed 
the migration activity of monolayer-cultured OA chondrocytes. Importantly, our study demonstrated that the migration is promoted by down-regulation of RECK with siRNA and the enhanced migration is canceled with MMP inhibitor BB94, suggesting that inhibition of chondrocyte migration by RECK is due to its MMP inhibitor activity. MT1-MMP and MMP-7, both of which are involved in cell surface or pericellular proteolysis ${ }^{55,56}$ and can be inhibited by RECK, ${ }^{6,7,9}$ are expressed by chondrocytes, including clustered chondrocytes in OA cartilage. ${ }^{15,57}$ Thus, these lines of evidence suggest the possibility that RECK functions as a negative regulator for chondrocyte mobilization by inhibition of MMP activity.

One other interesting finding in the present study is that RECK seems to be indispensable for the mitogen-induced proliferation of OA chondrocytes. Although RECK itself had no direct effects on chondrocyte proliferation, serum-, TGF$\beta$-, or IGF- $1+$ TGF- $\beta$-induced stimulation of the cell proliferation required the RECK expression. Because the effect of RECK silencing on proliferation was not rescued by BB94, GM6001, or TIMP-2, this seems to be independent of the MMP-inhibitor activity of RECK. RECK is a GPI-anchored protein without intracellular signaling domains, indicating that RECK itself cannot act as a growth factor receptor. On the other hand, integrin-mediated adhesion signals are known to collaborate or cross-talk with growth signaling of various growth factor receptors. ${ }^{32,58}$ In fact, FAK and ERK are thought to be two major components of signaling cascades linking cell-matrix interaction and proliferation. ${ }^{32}$ In the present study, we showed that phosphorylation of FAK and ERK is suppressed in RECK-silenced chondrocytes. Altogether it is plausible to think that RECK positively regulates growth factor signalings in OA chondrocytes through modulation of the functions of growth factor receptors or interactions with growth factors.

In summary, our data in the present study have demonstrated that RECK is overexpressed mainly in cloning chondrocytes in OA cartilage and RECK favors growth factorinduced proliferation of chondrocytes but suppresses their migration. Cloning of chondrocytes is thought to be formed by chondrocyte proliferation with limited migration and represent an incomplete repair process. ${ }^{3,4}$ Chondrocytes are endowed with the ability to proliferate in response to growth factors such as IGF-1 and TGF- $\beta$ as shown in the present and previous studies. ${ }^{29,30}$ Thus, one of the major obstacles of cartilage repair is considered to be defective migration of chondrocytes. ${ }^{59}$ If regrown chondrocytes could migrate appropriately to repopulate the damaged articular cartilage, more functional cartilage repair might be a result. Therefore, timely modulation of RECK expression during regenerative process of OA cartilage could contribute to improving repair by tissue engineering or reparative medicine.

\section{Acknowledgments}

We thank Ms. Yuko Hashimoto, Ms. Mayumi Kishi, and Ms. Michiko Uchiyama for their technical assistance. We are also grateful to Dr. Edward D. Harris, Jr. (Stanford University, School of Medicine) for reviewing the article.

\section{References}

1. Okada Y: Proteinases and matrix degradation. Kelley's Textbook of Rheumatology, ed 8. Edited by Firestein GS, Budd RC, Harris ED Jr, Mclnnes IB, Ruddy S, Sergent JS. Philadelphia, Saunders Elsevier, 2009, pp 115-134

2. Mankin HJ, Dorfman H, Lippiello L, Zarins A: Biochemical and metabolic abnormalities in articular cartilage from osteo-arthritic human hips. II Correlation of morphology with biochemical and metabolic data J Bone Joint Surg Am 1971, 53:523-537

3. Poole CA: Articular cartilage chondrons: form, function and failure. J Anat 1997, 191(Pt 1):1-13

4. Quintavalla J, Kumar C, Daouti S, Slosberg E, Uziel-Fusi S: Chondrocyte cluster formation in agarose cultures as a functional assay to identify genes expressed in osteoarthritis. J Cell Physiol 2005, 204:560-566

5. Takahashi C, Sheng Z, Horan TP, Kitayama H, Maki M, Hitomi K, Kitaura Y, Takai S, Sasahara RM, Horimoto A, Ikawa Y, Ratzkin BJ, Arakawa T, Noda M: Regulation of matrix metalloproteinase-9 and inhibition of tumor invasion by the membrane-anchored glycoprotein RECK. Proc Natl Acad Sci USA 1998, 95:13221-13226

6. Oh J, Takahashi R, Kondo S, Mizoguchi A, Adachi E, Sasahara RM, Nishimura S, Imamura Y, Kitayama H, Alexander DB, Ide C, Horan TP, Arakawa T, Yoshida H, Nishikawa S, Itoh $Y$, Seiki M, Itohara S, Takahashi C, Noda M: The membrane-anchored MMP inhibitor RECK is a key regulator of extracellular matrix integrity and angiogenesis. Cell 2001, 107:789-800

7. Miki T, Takegami Y, Okawa K, Muraguchi T, Noda M, Takahashi C: The reversion-inducing cysteine-rich protein with Kazal motifs (RECK) interacts with membrane type 1 matrix metalloproteinase and CD13/ aminopeptidase $\mathrm{N}$ and modulates their endocytic pathways. J Biol Chem 2007, 282:12341-12352

8. Muraguchi T, Takegami Y, Ohtsuka T, Kitajima S, Chandana EP Omura A, Miki T, Takahashi R, Matsumoto N, Ludwig A, Noda M, Takahashi C: RECK modulates Notch signaling during cortical neurogenesis by regulating ADAM10 activity. Nat Neurosci 2007, 10: $838-845$

9. Omura A, Matsuzaki T, Mio K, Ogura T, Yamamoto M, Fujita A, Okawa K, Kitayama H, Takahashi C, Sato C, Noda M: RECK forms cowbellshaped dimers and inhibits matrix metalloproteinase-catalyzed cleavage of fibronectin. J Biol Chem 2009, 284:3461-3469

10. Leco KJ, Waterhouse P, Sanchez OH, Gowing KL, Poole AR, Wakeham A, Mak TW, Khokha R: Spontaneous air space enlargement in the lungs of mice lacking tissue inhibitor of metalloproteinases-3 (TIMP-3). J Clin Invest 2001, 108:817-829

11. Soloway PD, Alexander CM, Werb Z, Jaenisch R: Targeted mutagenesis of Timp-1 reveals that lung tumor invasion is influenced by Timp-1 genotype of the tumor but not by that of the host. Oncogene 1996, 13:2307-2314

12. Wang $Z$, Juttermann R, Soloway PD: TIMP-2 is required for efficient activation of proMMP-2 in vivo. J Biol Chem 2000, 275:26411-26415

13. Kondo S, Shukunami C, Morioka Y, Matsumoto N, Takahashi R, Oh J, Atsumi T, Umezawa A, Kudo A, Kitayama H, Hiraki Y, Noda M: Dual effects of the membrane-anchored MMP regulator RECK on chondrogenic differentiation of ATDC5 cells. J Cell Sci 2007, 120:849-857

14. Altman R, Asch E, Bloch D, Bole G, Borenstein D, Brandt K, Christy W, Cooke TD, Greenwald R, Hochberg M, Howell D, Kaplan D, Koopman W, Longley S III, Mankin H, McShane DJ, Medsger T Jr, Meenan R, Mikkelsen W, Moskowitz R, Murphy W, Rothschild B, Segal M, Sokoloff L, Wolfe F: Development of criteria for the classification and reporting of osteoarthritis. Classification of osteoarthritis of the knee Diagnostic and Therapeutic Criteria Committee of the American Rheumatism Association. Arthritis Rheum 1986, 29:1039-1049

15. Fujita $Y$, Shiomi T, Yanagimoto $S$, Matsumoto $H$, Toyama $Y$, Okada $Y$ : Tetraspanin CD151 is expressed in osteoarthritic cartilage and is involved in pericellular activation of pro-matrix metalloproteinase 7 in osteoarthritic chondrocytes. Arthritis Rheum 2006, 54:3233-3243

16. Arnett FC, Edworthy SM, Bloch DA, McShane DJ, Fries JF, Cooper NS, Healey LA, Kaplan SR, Liang MH, Luthra HS, Medsger TA Jr, Mitchell DM, Neustadt DH, Pinals RS, Schaller JG, Sharp JT, Wilder RL, Hunder GG: The American Rheumatism Association 1987 revised criteria for the classification of rheumatoid arthritis. Arthritis Rheum 1988, 31:315-324

17. Kodama T, Ikeda E, Okada A, Ohtsuka T, Shimoda M, Shiomi T, 
Yoshida K, Nakada M, Ohuchi E, Okada Y: ADAM12 is selectively overexpressed in human glioblastomas and is associated with glioblastoma cell proliferation and shedding of heparin-binding epidermal growth factor. Am J Pathol 2004, 165:1743-1753

18. Mitsui $Y$, Mochizuki S, Kodama T, Shimoda M, Ohtsuka T, Shiomi T, Chijiiwa M, Ikeda T, Kitajima M, Okada Y: ADAM28 is overexpressed in human breast carcinomas: implications for carcinoma cell proliferation through cleavage of insulin-like growth factor binding protein-3. Cancer Res 2006, 66:9913-9920

19. Enomoto H, Inoki I, Komiya K, Shiomi T, Ikeda E, Obata K, Matsumoto $\mathrm{H}$, Toyama Y, Okada Y: Vascular endothelial growth factor isoforms and their receptors are expressed in human osteoarthritic cartilage. Am J Pathol 2003, 162:171-181

20. Liu LT, Peng JP, Chang HC, Hung WC: RECK is a target of EpsteinBarr virus latent membrane protein 1. Oncogene 2003, 22:8263-8270

21. Liu LT, Chang HC, Chiang LC, Hung WC: Histone deacetylase inhibitor up-regulates RECK to inhibit MMP-2 activation and cancer cell invasion. Cancer Res 2003, 63:3069-3072

22. Midwood KS, Salter DM: NG2/HMPG modulation of human articular chondrocyte adhesion to type $\mathrm{VI}$ collagen is lost in osteoarthritis. J Pathol 2001, 195:631-635

23. Oliver MH, Harrison NK, Bishop JE, Cole PJ, Laurent GJ: A rapid and convenient assay for counting cells cultured in microwell plates: application for assessment of growth factors. J Cell Sci 1989, 92 (Pt 3): 513-518

24. Jović M, Naslavsky N, Rapaport D, Horowitz M, Caplan S: EHD1 regulates beta1 integrin endosomal transport: effects on focal adhesions, cell spreading and migration. J Cell Sci 2007, 120:802-814

25. Khoshfetrat AB, Kino-Oka M, Takezawa Y, Yamamoto T, Sugawara K, Taya M: Seeding density modulates migration and morphology of rabbit chondrocytes cultured in collagen gels. Biotechnol Bioeng 2009, 102:294-302

26. Liang CC, Park AY, Guan JL: In vitro scratch assay: a convenient and inexpensive method for analysis of cell migration in vitro. Nat Protoc 2007, 2:329-333

27. Hamuro M, Polan J, Natarajan M, Mohan S: High glucose induced nuclear factor $\kappa \mathrm{B}$ mediated inhibition of endothelial cell migration. Atherosclerosis 2002, 162:277-287

28. Westhoff MA, Serrels B, Fincham VJ, Frame MC, Carragher NO SRC-mediated phosphorylation of focal adhesion kinase couples actin and adhesion dynamics to survival signaling. Mol Cell Biol 2004 24:8113-8133

29. Okada A, Mochizuki S, Yatabe T, Kimura T, Shiomi T, Fujita Y, Matsumoto H, Sehara-Fujisawa A, Iwamoto Y, Okada Y: ADAM-12 (meltrin $\alpha$ ) is involved in chondrocyte proliferation via cleavage of insulinlike growth factor binding protein 5 in osteoarthritic cartilage. Arthritis Rheum 2008, 58:778-789

30. Guerne PA, Sublet A, Lotz M: Growth factor responsiveness of human articular chondrocytes: distinct profiles in primary chondrocytes, subcultured chondrocytes, and fibroblasts. J Cell Physiol 1994, 158: 476-484

31. Wozniak MA, Modzelewska K, Kwong L, Keely PJ: Focal adhesion regulation of cell behavior. Biochim Biophys Acta 2004, 1692:103-119

32. Lee JW, Juliano R: Mitogenic signal transduction by integrin- and growth factor receptor-mediated pathways. Mol Cells 2004, 17:188-202

33. Echizenya M, Kondo S, Takahashi R, Oh J, Kawashima S, Kitayama H, Takahashi C, Noda M: The membrane-anchored MMP-regulator RECK is a target of myogenic regulatory factors. Oncogene 2005, 24:5850-5857

34. Nuttall RK, Sampieri CL, Pennington CJ, Gill SE, Schultz GA, Edwards DR: Expression analysis of the entire MMP and TIMP gene families during mouse tissue development. FEBS Lett 2004, 563:129-134

35. Noda M, Takahashi C: Recklessness as a hallmark of aggressive cancer. Cancer Sci 2007, 98:1659-1665

36. van Lent PL, Span PN, Sloetjes AW, Radstake TR, van Lieshout AW, Heuvel JJ, Sweep CG, van den Berg WB: Expression and localisation of the new metalloproteinase inhibitor RECK (reversion inducing cysteine-rich protein with Kazal motifs) in inflamed synovial membranes of patients with rheumatoid arthritis. Ann Rheum Dis 2005, 64 368-374

37. Hsu MC, Chang HC, Hung WC: HER-2/neu represses the metastasis suppressor RECK via ERK and Sp transcription factors to promote cell invasion. J Biol Chem 2006, 281:4718-4725

38. Sasahara RM, Takahashi C, Noda M: Involvement of the Sp1 site in ras-mediated downregulation of the RECK metastasis suppressor gene. Biochem Biophys Res Commun 1999, 264:668-675

39. Gabriely G, Wurdinger T, Kesari S, Esau CC, Burchard J, Linsley PS, Krichevsky AM: MicroRNA 21 promotes glioma invasion by targeting matrix metalloproteinase regulators. Mol Cell Biol 2008, 28:53695380

40. Zhang Z, Li Z, Gao C, Chen P, Chen J, Liu W, Xiao S, Lu H: miR-21 plays a pivotal role in gastric cancer pathogenesis and progression. Lab Invest 2008, 88:1358-1366

41. Vagima $Y$, Avigdor A, Goichberg P, Shivtiel S, Tesio M, Kalinkovich A, Golan K, Dar A, Kollet O, Petit I, Perl O, Rosenthal E, Resnick I, Hardan I, Gellman YN, Naor D, Nagler A, Lapidot T: MT1-MMP and RECK are involved in human $\mathrm{CD} 34^{+}$progenitor cell retention, egress, and mobilization. J Clin Invest 2009, 119:492-503

42. Oh J, Seo DW, Diaz T, Wei B, Ward Y, Ray JM, Morioka Y, Shi S, Kitayama H, Takahashi C, Noda M, Stetler-Stevenson WG: Tissue inhibitors of metalloproteinase 2 inhibits endothelial cell migration through increased expression of RECK. Cancer Res 2004, 64: 9062-9069

43. Oh J, Diaz T, Wei B, Chang H, Noda M, Stetler-Stevenson WG: TIMP-2 upregulates RECK expression via dephosphorylation of paxillin tyrosine residues 31 and 118. Oncogene 2006, 25:4230-4234

44. Middleton J, Manthey A, Tyler J: Insulin-like growth factor (IGF) receptor. IGF-I, interleukin-1 $\beta$ (IL-1 $\beta$ ), and IL-6 mRNA expression in osteoarthritic and normal human cartilage, J Histochem Cytochem 1996, 44:133-141

45. Moos V, Fickert S, Muller B, Weber U, Sieper J: Immunohistological analysis of cytokine expression in human osteoarthritic and healthy cartilage. J Rheumatol 1999, 26:870-879

46. Schlaak JF, Pfers I, Meyer Zum Buschenfelde KH, Marker-Hermann E: Different cytokine profiles in the synovial fluid of patients with osteoarthritis, rheumatoid arthritis and seronegative spondylarthropathies. Clin Exp Rheumatol 1996, 14:155-162

47. Morioka Y, Monypenny J, Matsuzaki T, Shi S, Alexander DB, Kitayama $\mathrm{H}$, Noda $\mathrm{M}$ : The membrane-anchored metalloproteinase regulator RECK stabilizes focal adhesions and anterior-posterior polarity in fibroblasts. Oncogene 2009, 28:1454-1464

48. DeMali KA, Wennerberg K, Burridge K: Integrin signaling to the actin cytoskeleton. Curr Opin Cell Biol 2003, 15:572-582

49. Aszodi A, Hunziker EB, Brakebusch C, Fassler R: Beta1 integrins regulate chondrocyte rotation. G1 progression, and cytokinesis, Genes Dev 2003, 17:2465-2479

50. Durrant LA, Archer CW, Benjamin M, Ralphs JR: Organisation of the chondrocyte cytoskeleton and its response to changing mechanical conditions in organ culture, J Anat 1999, 194(Pt 3):343-353

51. Morales TI: Chondrocyte moves: clever strategies? Osteoarthritis Cartilage 2007, 15:861-871

52. Kouri JB, Arguello C, Luna J, Mena R: Use of microscopical techniques in the study of human chondrocytes from osteoarthritic cartilage: an overview. Microsc Res Tech 1998, 40:22-36

53. Kouri JB, Jimenez SA, Quintero M, Chico A: Ultrastructural study of chondrocytes from fibrillated and non-fibrillated human osteoarthritic cartilage. Osteoarthritis Cartilage 1996, 4:111-125

54. Frenkel SR, Clancy RM, Ricci JL, Di Cesare PE, Rediske JJ, Abramson SB: Effects of nitric oxide on chondrocyte migration, adhesion, and cytoskeletal assembly. Arthritis Rheum 1996, 39:1905-1912

55. Sato H, Takino T, Okada Y, Cao J, Shinagawa A, Yamamoto E, Seiki M: A matrix metalloproteinase expressed on the surface of invasive tumour cells. Nature 1994, 370:61-65

56. Shiomi T, Inoki I, Kataoka F, Ohtsuka T, Hashimoto G, Nemori R, Okada Y: Pericellular activation of proMMP-7 (promatrilysin-1) through interaction with CD151. Lab Invest 2005, 85:1489-1506

57. Imai K, Ohta S, Matsumoto T, Fujimoto N, Sato H, Seiki M, Okada Y: Expression of membrane-type 1 matrix metalloproteinase and activation of progelatinase $\mathrm{A}$ in human osteoarthritic cartilage. Am J Pathol 1997, 151:245-256

58. Miyamoto S, Teramoto H, Gutkind JS, Yamada KM: Integrins can collaborate with growth factors for phosphorylation of receptor tyrosine kinases and MAP kinase activation: roles of integrin aggregation and occupancy of receptors. J Cell Biol 1996, 135:1633-1642

59. Khan IM, Gilbert SJ, Singhrao SK, Duance VC, Archer CW: Cartilage integration: evaluation of the reasons for failure of integration during cartilage repair. A review. Eur Cell Mater 2008, 16:26-39 